\title{
Role of Self-Compassion and Sense of Coherence and Thought Action Fusion in Prediction of Psychological Well-Being among Post-Traumatic Stress Disorder Veterans
}

\section{ART ICLE INF O}

\section{Article Type}

Descriptive Study

\section{Authors}

Mikaeili N. ${ }^{1} P h D$,

Einy $\mathrm{S}{ }^{*} M S C$,

Tagavy R. ${ }^{2} M S C$

How to cite this article
Mikaeili N, Einy S, Tagavy R. Role
of Self-Compassion and Sence of
Co-herence and Thought Action
Fusion in Prediction of Psychol-
ogical Well-Being among Post-
Traumatic Stress Disorder Veter-
ans. Iranian Journal of War \& Pu-
blic Health. 2017;9 (1):33-39.

*Psychology Department, Psychology \& Educational Sciences Faculty, University of Mohaghegh Ardabili, Ardabil, Iran

${ }^{1}$ Psychology Department, Psychology and Educational Sciences Faculty, University of Mohaghegh Ardabili, Ardabil, Iran

${ }^{2}$ Psychology Department, Psychology Faculty, Ardabil Branch, Islamic Azad University, Ardabil, Iran

\section{Correspondence}

Address: Psychology and Educational Sciences Faculty, End of Daneshgah Street, University of Mohaghegh Ardabili, Ardabil, Iran

Phone: +98 (45) 33262678

Fax: +98 (45) 33511508 sanaz.einy@yahoo.com

\section{Article History}

Received: November 18, 2016

Accepted: January 7, 2017

ePublished: March 10, 2017

\section{A B S T R A C T}

Aims Due to low psychological well-being level in veterans with the post-traumatic stress disorders, the symptoms are exacerbated in them. The aim of the study was to predict the psychological well-being in the veterans with post-traumatic stress disorders, based on the personality parameters including self-compassion, sense of coherence, and the thought-action fusion.

Instrument \& Methods In the descriptive correlational study, 120 veterans with posttraumatic stress disorders, hospitalized in Isar Psychiatric Hospital in Ardabil, were studied as available subjects in 2016. Data was collected using the psychological well-being questionnaire, self-compassion scale, the sense of coherence scale, and the revised thought-action fusion scale. Data was analyzed by SPSS 17 software using Pearson correlation coefficient test and step-wise regression analysis.

Findings The psychological well-being in the veterans with post-traumatic stress disorders significantly and positively correlated with the sense of coherence and the self-compassion, while it significantly and negatively correlated with the thought-action fusion $(p<0.05)$. In addition, $63 \%, 49 \%$, and $67 \%$ of variance of psychological well-being scores in the veterans with post-traumatic stress disorders were predicted by the sense of coherence, thought-action fusion, and the self-compassion, respectively $(\mathrm{p}<0.001)$.

Conclusion High sense of coherence and high self-compassion, as well as low thought-action fusion, improve psychological well-being in the veterans with post-traumatic stress disorders.

Keywords Sense of Coherence; Compassion; Thought-Action Fusion; Health; Stress Disorder, Post-Traumatic; Veterans

\section{CIT A T ION LINKS}

[1] Diagnostic and statistical manual of mental disorders ... [2] Psychological hcalth status of mustard gas ... [3] Post-traumatic stress disorder in male chemical ... [4] Predictors of decline in overall mental health... [5] Minimally adequate mental health care and latent ... [6] The relationship between religious identity... [7] The measurement of health: Concepts and indicators ... [8] Effectiveness of training based on virginia stair's communicative approach on parent ... [9] Pain-related musculoskeletal disorders ... [10] Self-compassion: An alternative conceptualization ... [11] Self-compassion and adaptive psychological ... [12] Compassionate mind training for people with high ... [13] Fear of self-compassion and psychological ... [14] The indirect effect of social support on posttrauma ... [15] Bluemke M. Through the tunnel ... [16] Correlation between psychological well-being ... [17] Why is Antonovskys sense of coherence not correlated to physical health? Analysing ...[18] Is parental sense of coherence associated with child ... [19] The structure and properties of the sense of coherence ... [20] Post-traumatic cognitions as a mediator of the relationship between sense of coherence and post-traumatic stress disorder ... [21] Thought-action fusion: Review of the literature and future ... [22] Frome Intrusive to oscillating ... [23] The thought-action fusion scale: Further evidence for its reliability ... [24] Understanding the relationship between posttraumatic stress disorder and trauma cognitions ... [25] The effects of familiarity on thought --action ... [26] Effects of selective serotonin reuptake inhibitors ... [27] Childhood trauma and thought action fusion: A multi-method ... [28] Happiness is everything is it? Exploration on the meaning of psychological... [29] Reliability and validity of ryff's psychological well-being ... [30] Psychometric properties of the Persian version of the self compassion scale ... [31] The structure and properties of the sense of coherence ... [32] Validation of Sense of Coherence (SOC) ... [33] Thought-action fusion in obsessive- compulsive... [34] Psychometric properties of revised thought-action fusion questionnaire ... 
مواجهه با حوادث آسيبزاى زندگى يِيدا مىشود؛ فرد باصورت

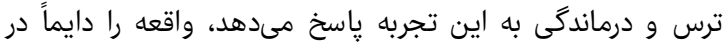

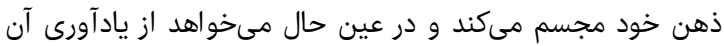

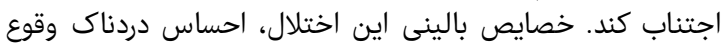

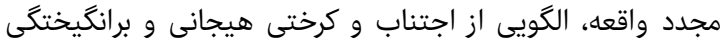

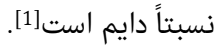

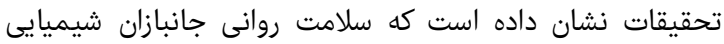

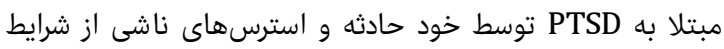

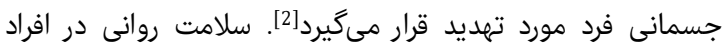

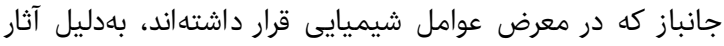

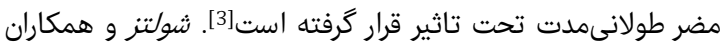

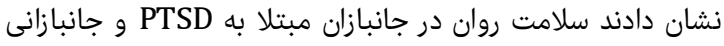

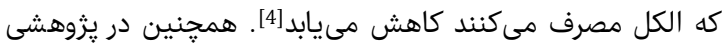

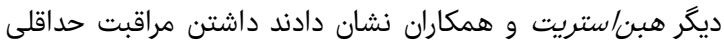

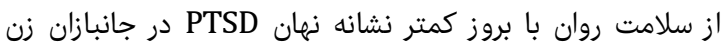

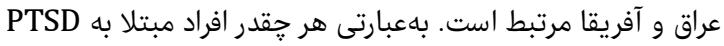

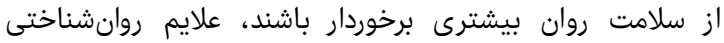

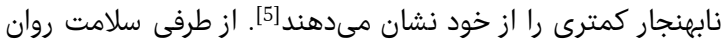

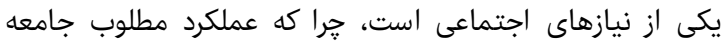

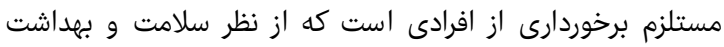

روانى در وضعيت مطلوبى قرار دارند [6].

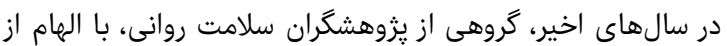

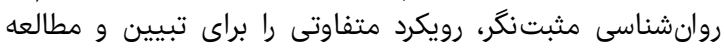

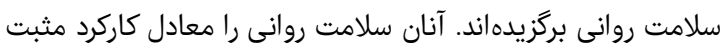

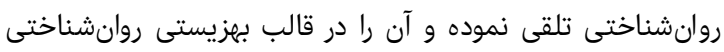

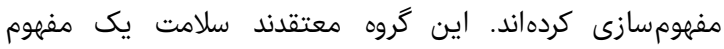

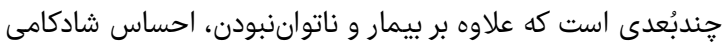

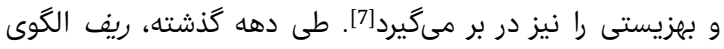

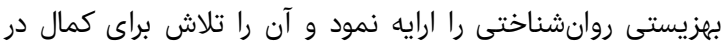

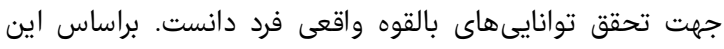

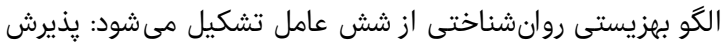

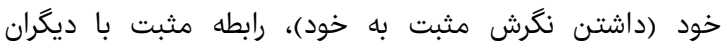

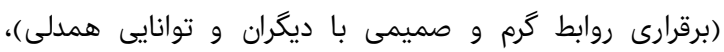

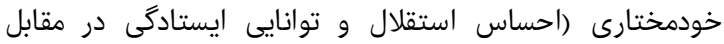

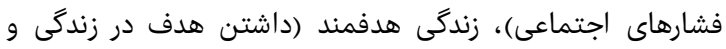

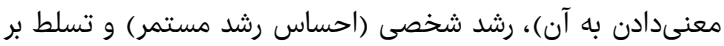

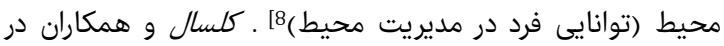

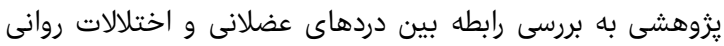

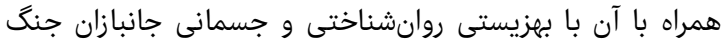

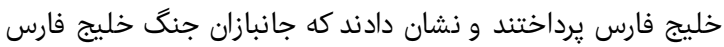

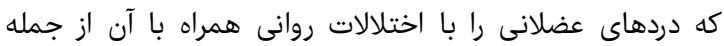

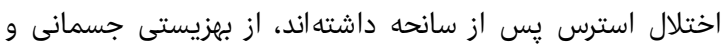

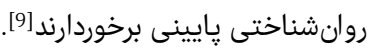

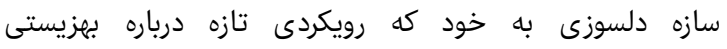

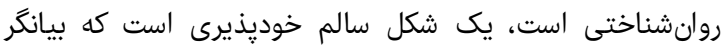

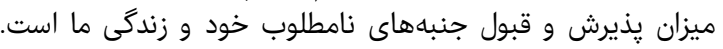

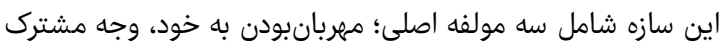

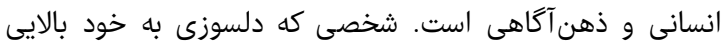

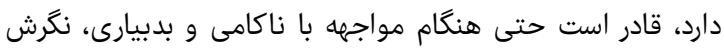

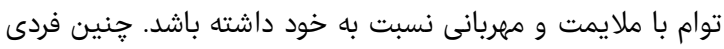

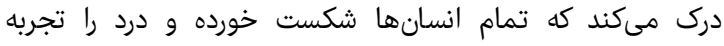

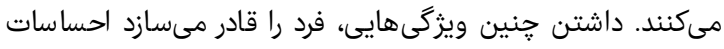

دوره ، شماره ا، زمستان هوس|
نقش دلسوزى به خود و و حس انسجام و آميختىى

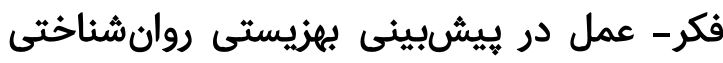

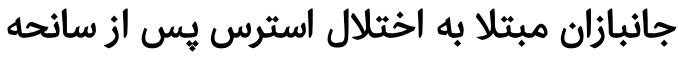

PhD نيلوفر ميكاييلى

كروه روان شناسى، دانشكده علومتربيتى و روان شناسى، دانشكاه محقق اردبيلى،

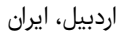

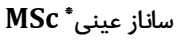

كروه روان شناسى، دانشكده علومتربيتى و روانشناسى، دانشگاه محقق اردبيلى،

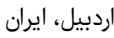

رامين تقوى إيران

كروه روانشناسى، دانشكده روانشناسى، واحد اردبيل، دانشكاه آزاد اسلامى،

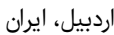

جكيده

اهداف: در جانبازان مبتلا به اختلال استرس يس إن از سانحه سطح بهزيستى

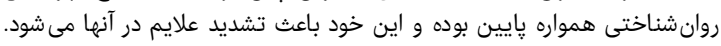

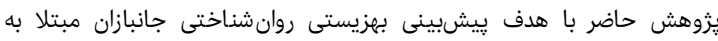

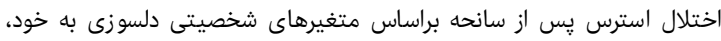

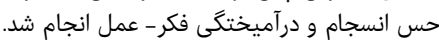

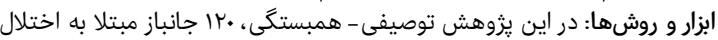

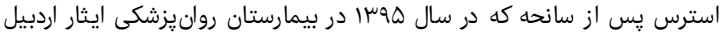

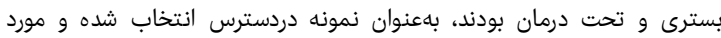
برارسى قرار گرفتند

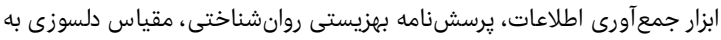

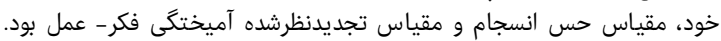

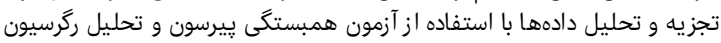

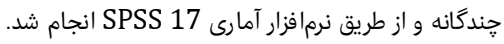

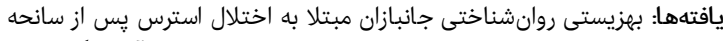

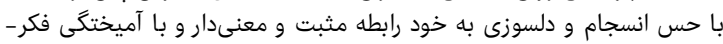

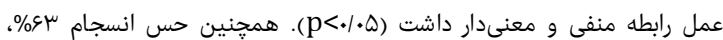

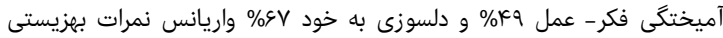

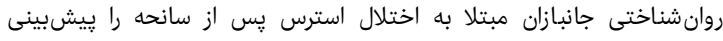

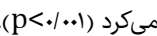

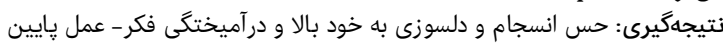

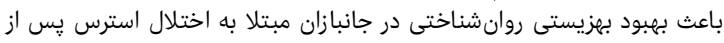

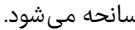

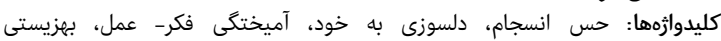
روان شناختى، اختلال استرس يس إز سانحه، جانباز

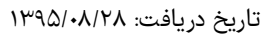

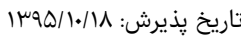
"نويسنده مسئول: sanaz.einy@yahoo.com

بروز جنگ، جزء جدايىنايذير زندگى آدمى بوده است. از عوارض هر

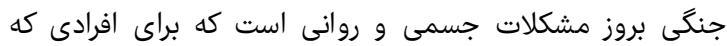

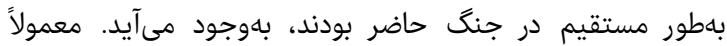

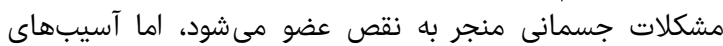

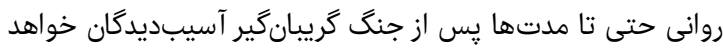

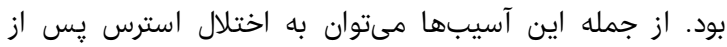

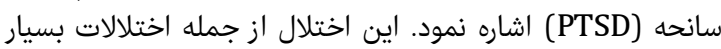

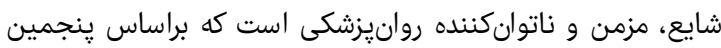

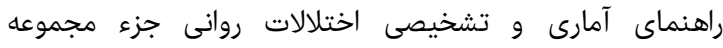

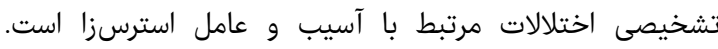
عبارت است از مجموعهاى از علايم نوعى سندروم كه در يى آسي استر

فصل نامه علمى - يُوهشى طب جانباز 


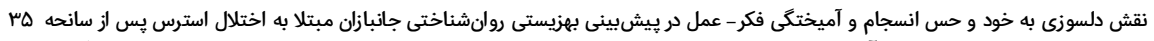

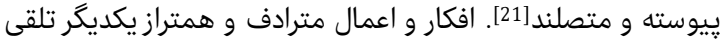

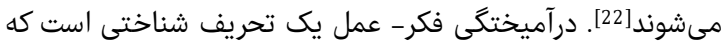

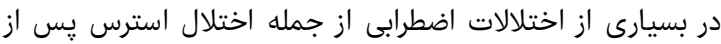

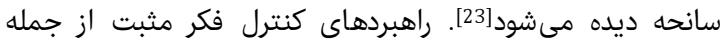

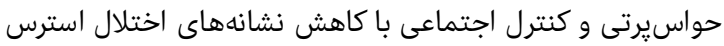

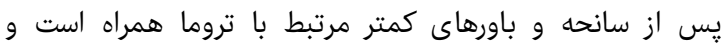

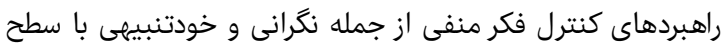

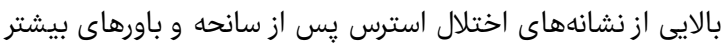

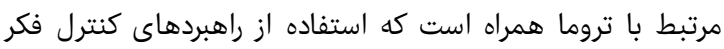

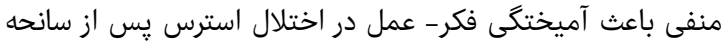

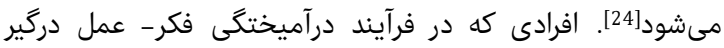

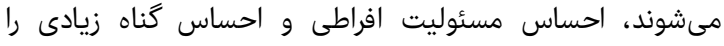

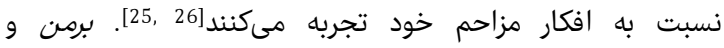

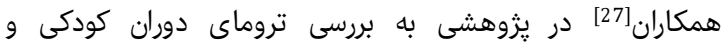

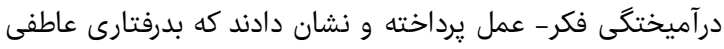

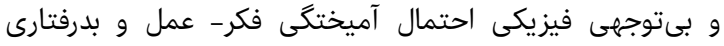

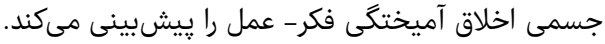

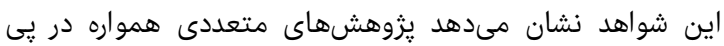

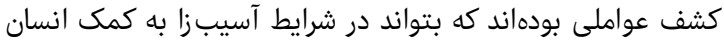

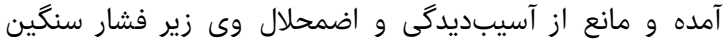

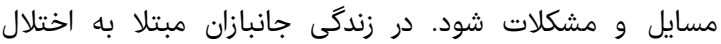

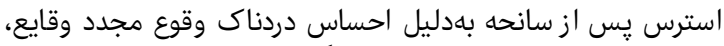

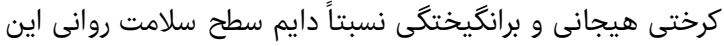

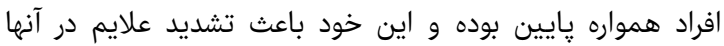

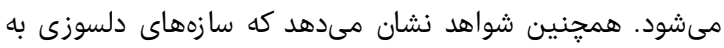
خود و حس انسجام، دو مورد از متغيرهاى مران مرتبط بان با بهزيستى

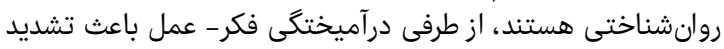

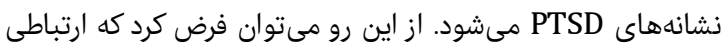

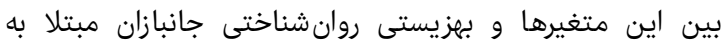

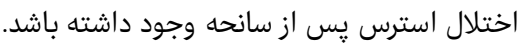

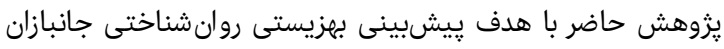

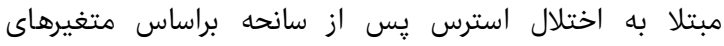

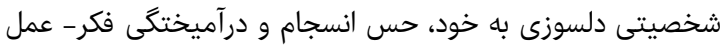

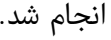

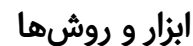

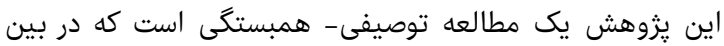

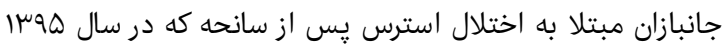

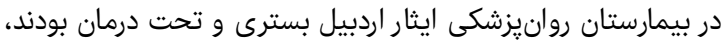

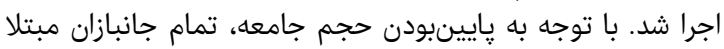

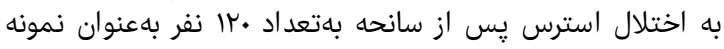

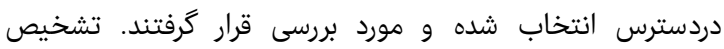

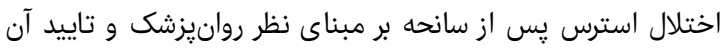

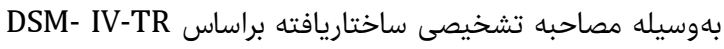

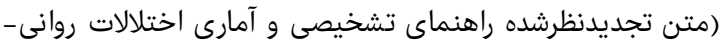

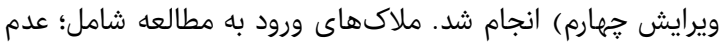

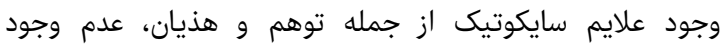

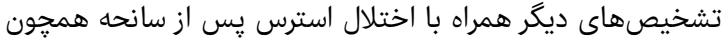

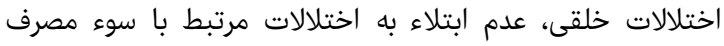

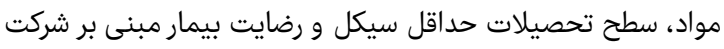

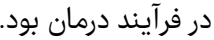
از ₹ ابزار براى جمع آورى اطلاعات استفاده شد:
و هيجان هاى خود را بهطور مناسب تجربه نمايد، بدون اينكه آنها را

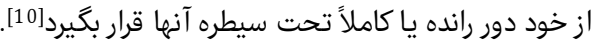

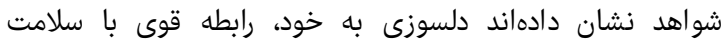

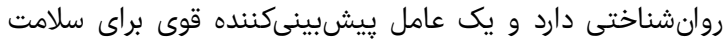

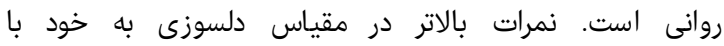

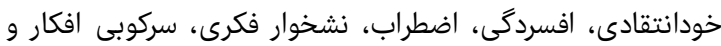

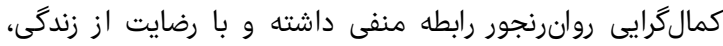

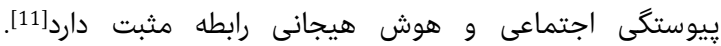

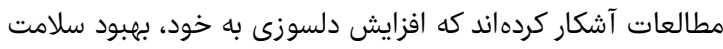

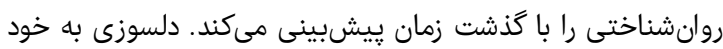

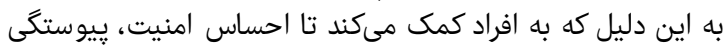

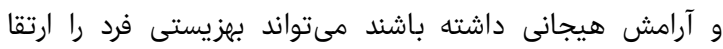

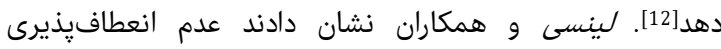

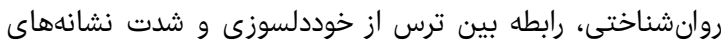

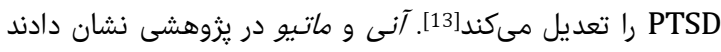

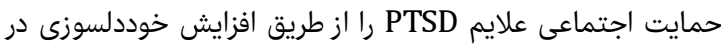

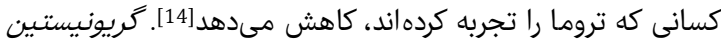

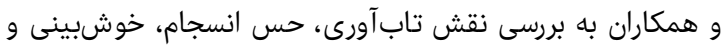

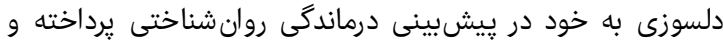

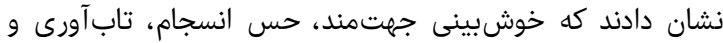

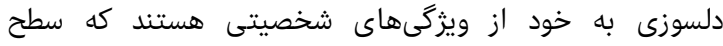

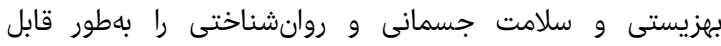

ملاحظهاى ارتقا مىدهند [15].

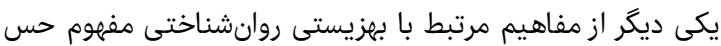

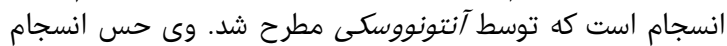

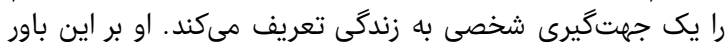

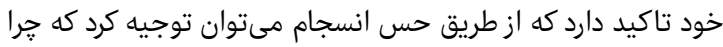

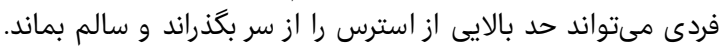

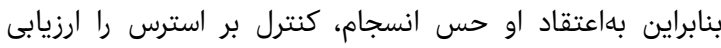

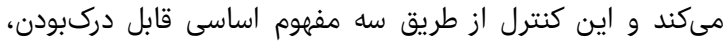

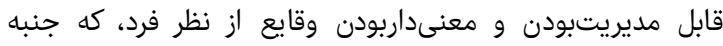

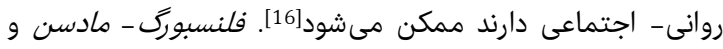

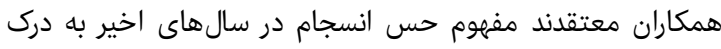

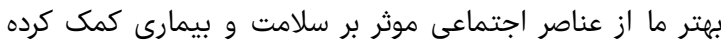

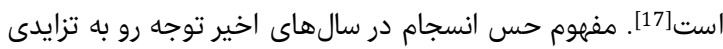
را زير عنوان يك مدل سلامتىزا به خود جلب كرد [18]. آنتونووسكى رونى

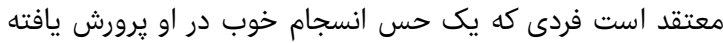

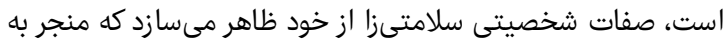

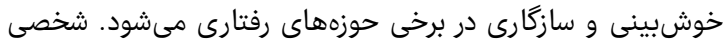

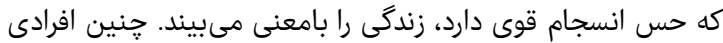

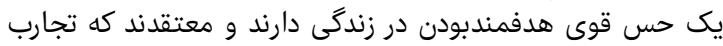

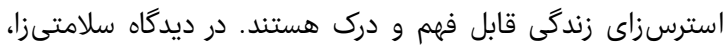

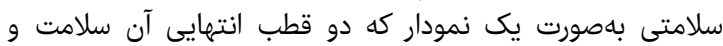

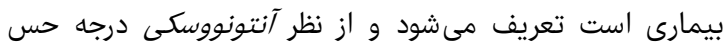

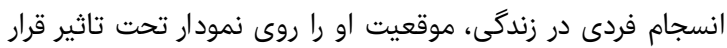

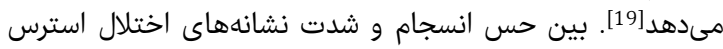

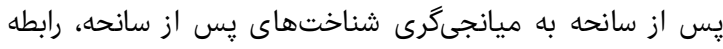
منفى معنىدارى وجود دارد[20].

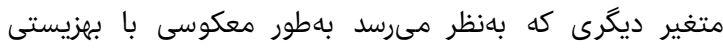

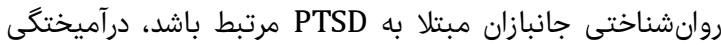

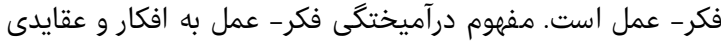
اشاره دارد كه در آن افكار و عمل بهُطور غيرقابل درابل تفكيكى به يكديگر 
مقياس ينجدرجهاى ليكرت از صفر (كاملاً مخالف) تا ع (كاملاً

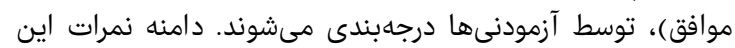

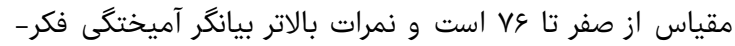

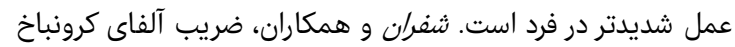

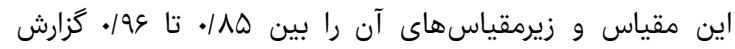

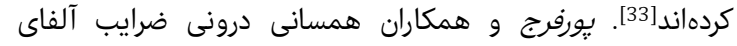

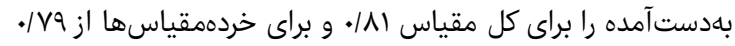

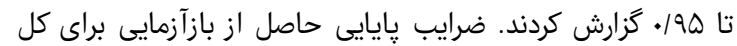

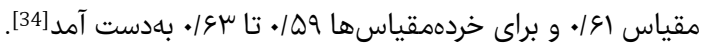

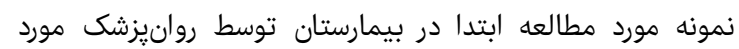

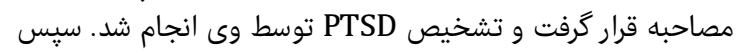

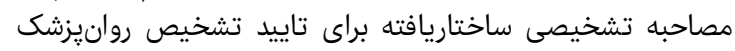

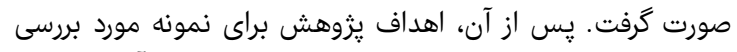

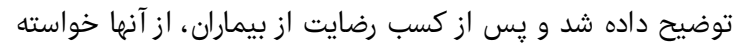

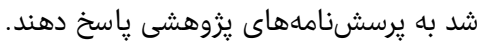

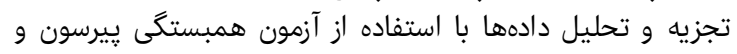

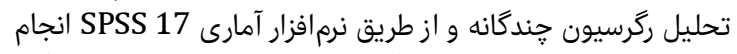

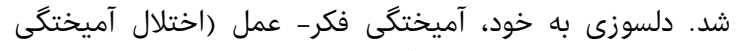

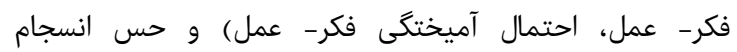

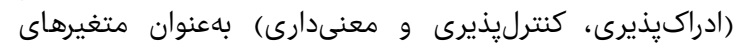

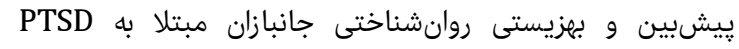

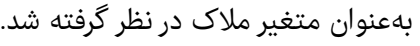

\section{يافتهها}

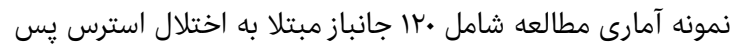

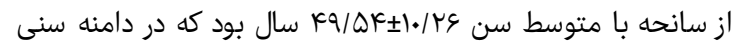

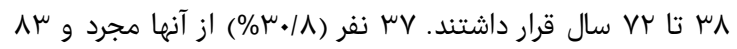

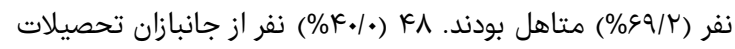

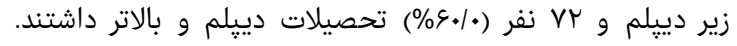

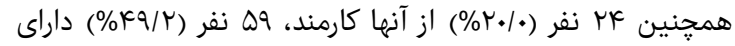

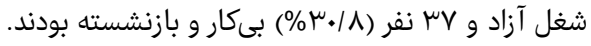

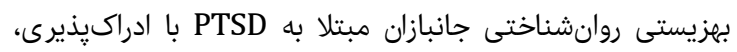

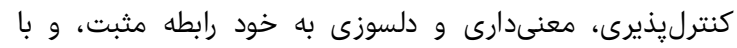

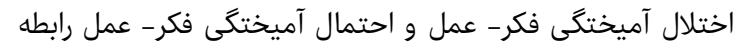

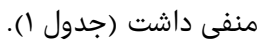

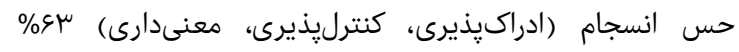

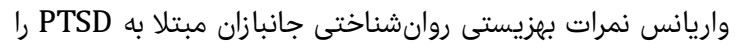

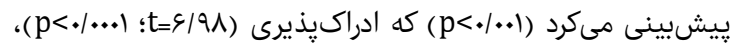

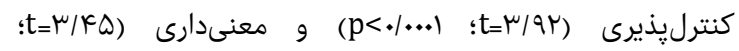

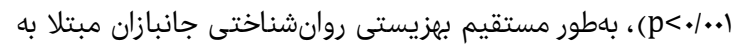

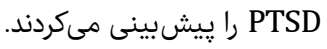

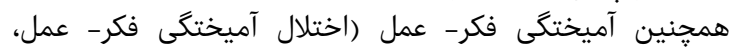

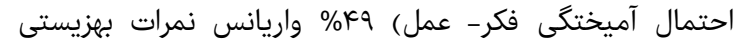

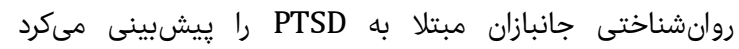
و (p<>/..1)

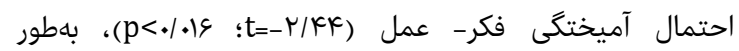

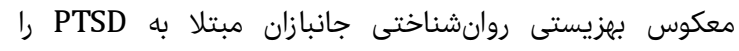
ييشبينى مىكردند.

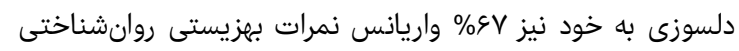

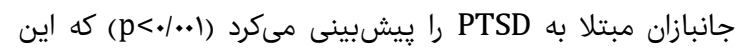

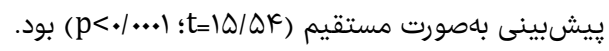

1- مقياس بهزيستى روانشناختى: اين مقياس كه توسط كارول

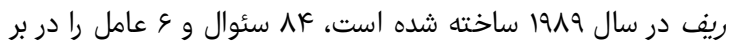

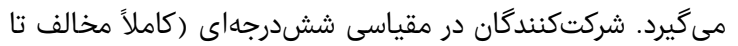

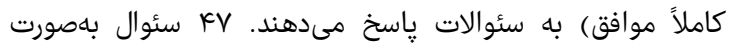

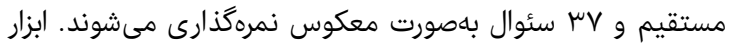

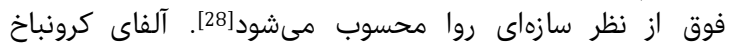

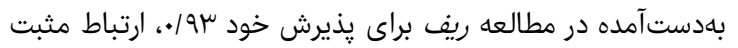

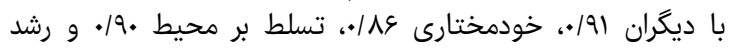

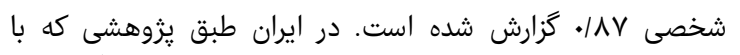

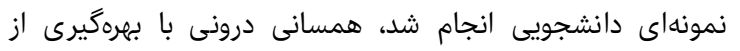

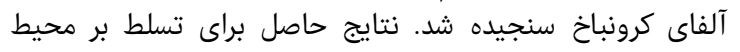

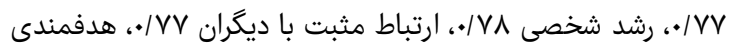

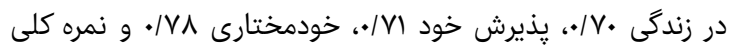

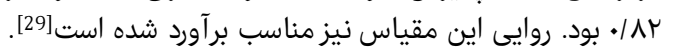

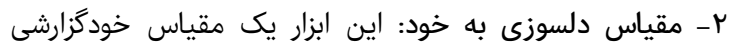

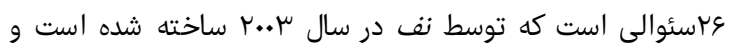

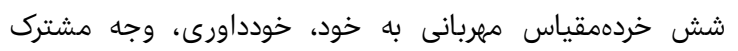

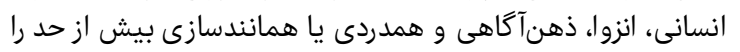

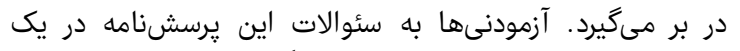

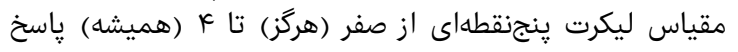

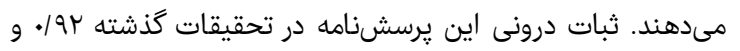

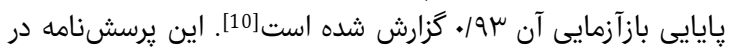

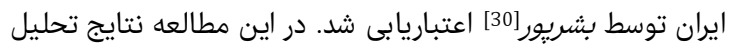

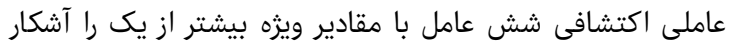

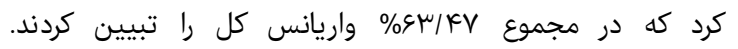

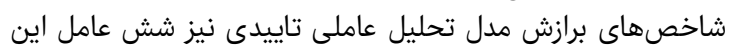

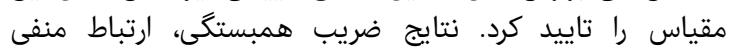

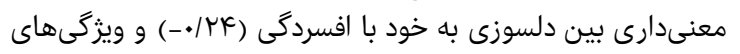

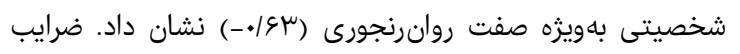
آلفاى كرونباخ خردهمقياسها و نمره كلى مقياس نيز ديز در دامنه هواء.

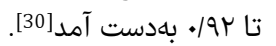

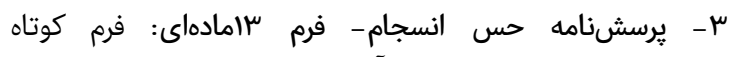

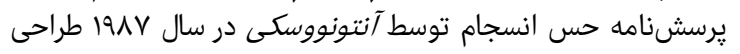

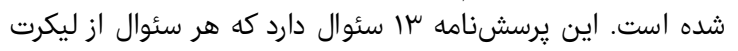

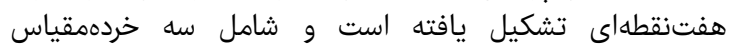

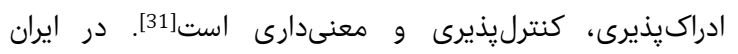

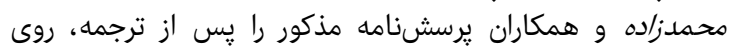

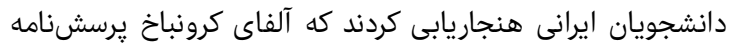

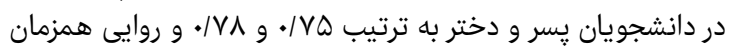

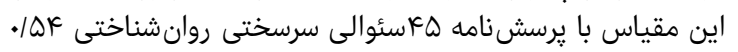

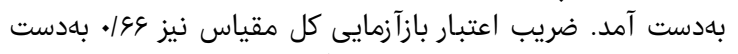

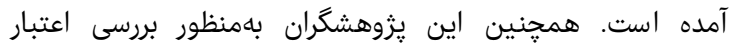

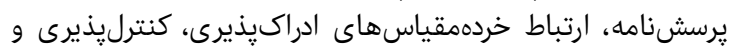

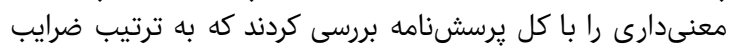

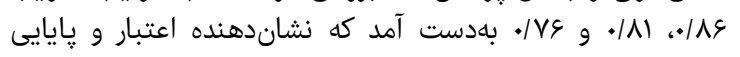

مطلوب مقياس است ف [32].

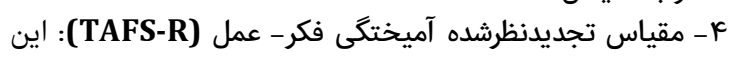

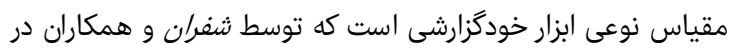

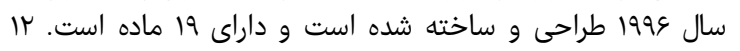

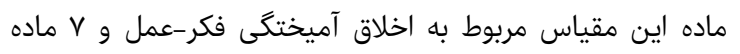

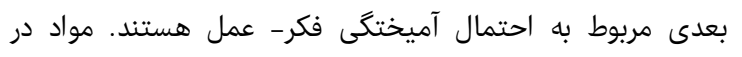




\begin{tabular}{|c|c|c|c|c|c|c|c|}
\hline 8 & $\Delta$ & F & r & r & 1 & ميانگين آمارى & متغيرها \\
\hline & & & & & 1 & $\mid V / \mu \omega \pm \Delta / \Delta$ & 1- ادراكيذيرى \\
\hline & & & & 1 & $* . / F \mu$ & $1 \cdot /|\omega \pm \mu / \Lambda|$ & r-كنترل يذيرى \\
\hline & & & 1 & $* / 4 q$ & $* * . \mid Q 1$ & $\mid \& / \Lambda \Delta \pm \Delta / r V$ & " - معنى - مارى \\
\hline & & 1 & $*_{-} / \Delta r$ & $*_{-\bullet / F \mu}$ & $*_{-} \cdot \varepsilon \mu$ & $r r / \varepsilon \cdot \pm \varepsilon / r q$ & F- اختلال آميختكى فكر- عمل \\
\hline & 1 & $* * . / \Delta s$ & $*_{-} / \Delta r$ & $*_{-\bullet / K G}$ & $* * \cdot / \Delta r$ & $\|H / V \Delta \pm F /\|$ & هـ - احتمال آميختخى فكر - عمل \\
\hline 1 & $*_{-}^{*} / \Delta F$ & $*_{-} \cdot \mid \& \mathrm{~V}$ & $.19 \Lambda$ & *. & $* / V k$ & $V \wedge / r \cdot \pm 1 r / \mu^{\mu} V$ & צ- دلسوزى به خود \\
\hline *./Ar & $*+. / 01$ & $*_{-} .19 \Lambda$ & $* * .|8|$ & $* * .101$ & $* * . / \mathrm{VI}$ & $r \varepsilon q / r r \pm r q / V k$ & V - V - Vزيستى روان شناختى \\
\hline
\end{tabular}

دارد. براساس نظريه نف [10] مىتوان حنين تبيين كرد كه جانبازان

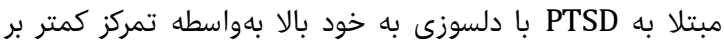

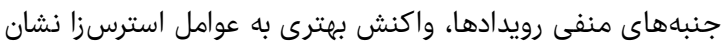

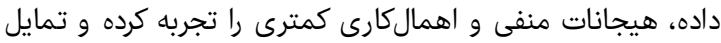

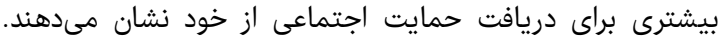

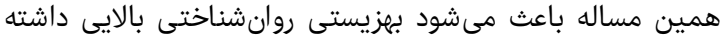

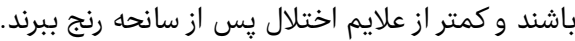

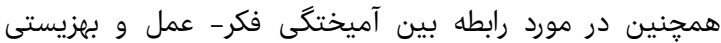

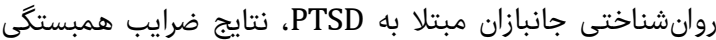

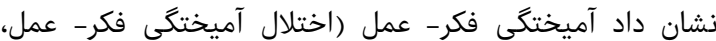

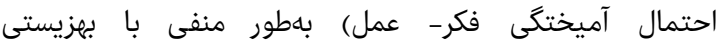

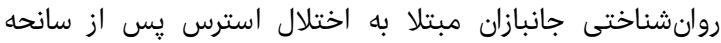

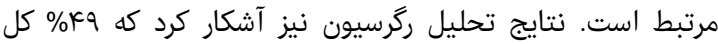

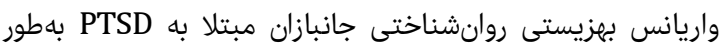

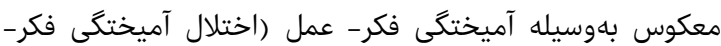

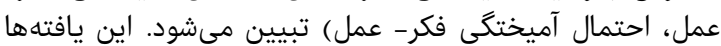

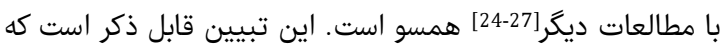

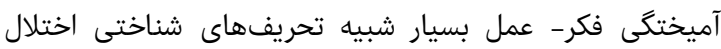

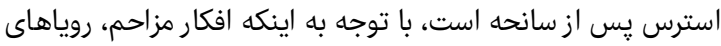

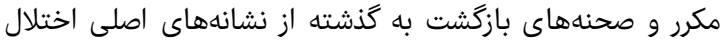

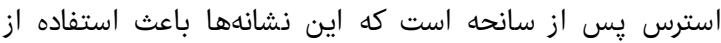

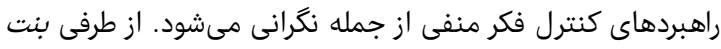

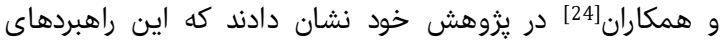

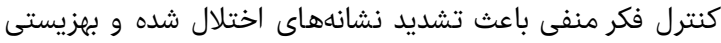

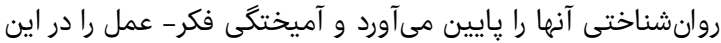

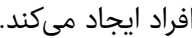

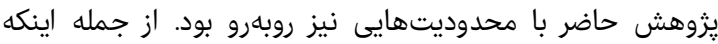

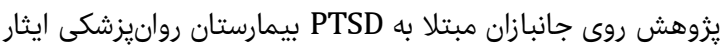

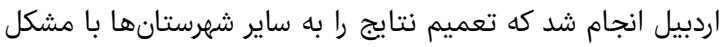

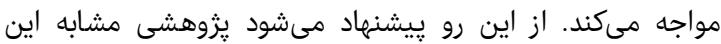

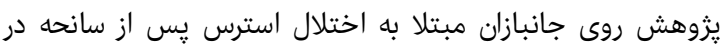

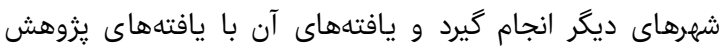

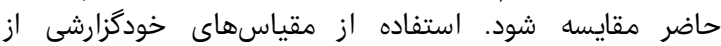

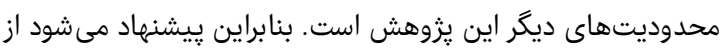

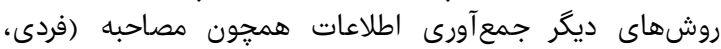

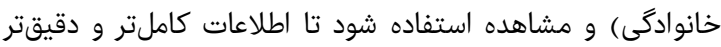

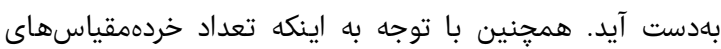

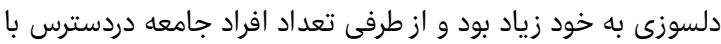

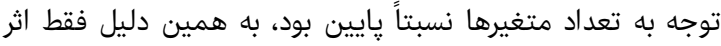

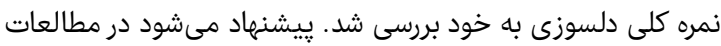

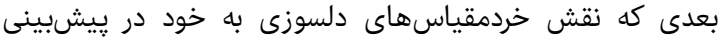

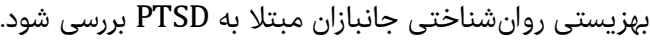

يززوهش حاضر با هدف يِيشبينى بهزيستى روانشناختى جانبازان

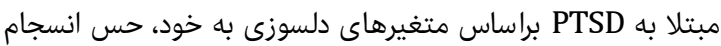

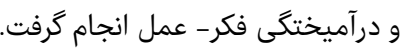

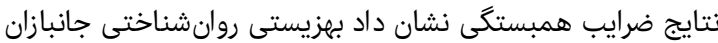

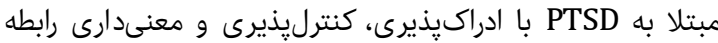

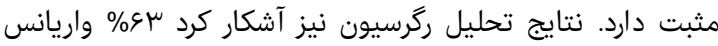

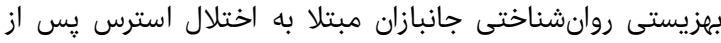

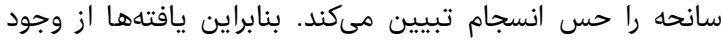

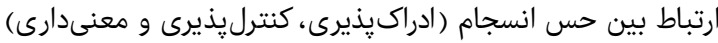

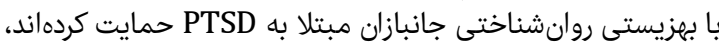

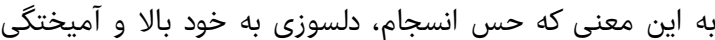
فكر- عمل يايين با بجزيستى روان شناختى بيشتر در جانبازان انبان مبتلا

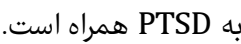

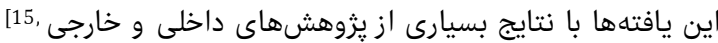

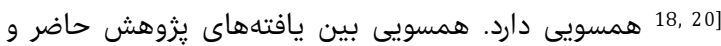

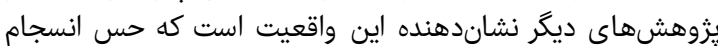

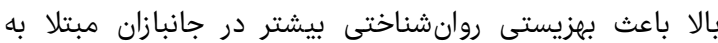

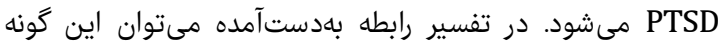

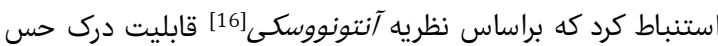

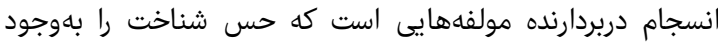

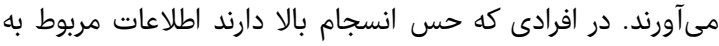

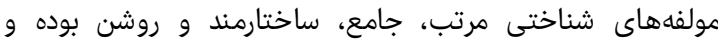

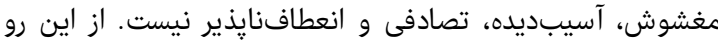

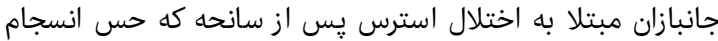

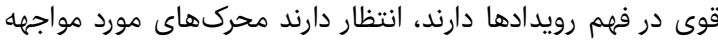

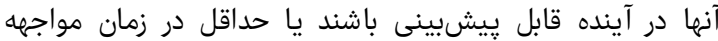

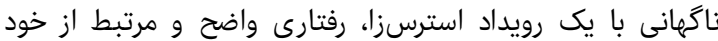

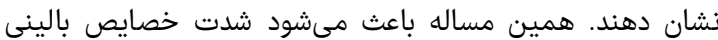

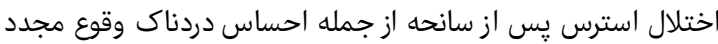

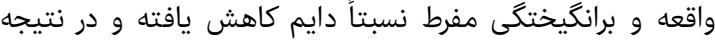

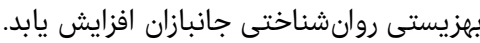

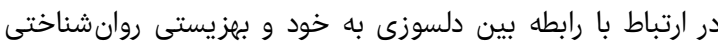

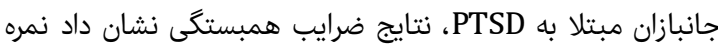

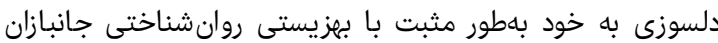

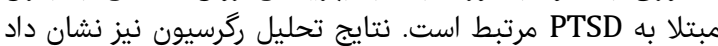

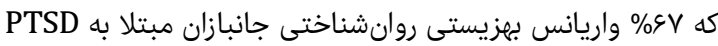

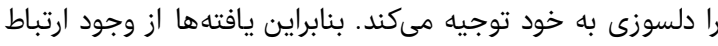

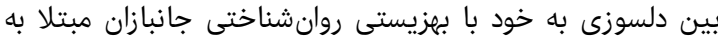

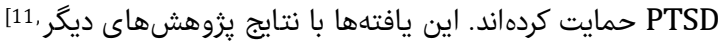

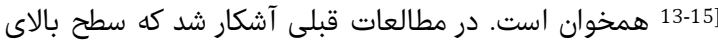
دلسوزى به خود با سطح بالاى كاركرد بهتر روانشناختى آشار ارتباط بال 
12- Gilbert P, Proctor S. Compassionate mind training for people with high shame and self-criticism: Overview and pilot study of a group therapy approach. Clin Psychol Psychother. 2006;13(6):353-79.

13- Lynsey RM, Andrew MS, Holly KO. Fear of selfcompassion and psychological inflexibility interact to predict PTSD symptom severity. J Context Behav Sci. 2015;4(1):37-41.

14- Maheux A, Price M. The indirect effect of social support on post-trauma psychopathology via selfcompassion. Personal Individ Differ. 2016;88:102-7.

15- Grevenstein D, Aguilar-Raab C, Schweitzer J, Bluemke M. Through the tunnel, to the light: Why sense of coherence covers and exceeds resilience, optimism, and self-compassion. Personal Individ Differ. 2016;98:20817.

16- Aghayousefi AR, Sharif N. Correlation between psychological well-being and sense of coherence in the students of Islamic Azad University-Central Tehran Branch. Pejouhandeh. 2011;15(6):273-9. [Persian] 17- Flensborg-Madsen T, Vetegodt S, Merrick J. Why is Antonovskys sense of coherence not correlated to physical health? Analysing Antonovsky's 29-item sense of coherence scale (SOC-29). Sci World J. 2005;5:767-76.

18- Groholt EH, Stigum H, Nordhagen R, Kohler L. Is parental sense of coherence associated with child health?. Eur J Public Health. 2003;13(3):195-201.

19- Antonovsky A. The structure and properties of the sense of coherence scale. Soc Sci Med. 1993;36(6):72533.

20- Kazmierczak I, Strelau J, Zawadzki B. Post-traumatic cognitions as a mediator of the relationship between sense of coherence and post-traumatic stress disorder after motor vehicle accidents. Transp Res Part F Traffic Psychol Behav. 2016;41:38-44.

21- Berle D, Starcevic V. Thought-action fusion: Review of the literature and future directions. Clin Psychol Rev. 2005;25(3):263-84.

22- Pierce AG. Frome Intrusive to oscillating Thought. Arch Psychiatr Nurs. 2007;21(5):278-86.

23- Rassin E, Merckelbach H, Muris P, Schmidt H. The thought-action fusion scale: Further evidence for its reliability and validity. Behav Res Ther. 2001;39(5):53744.

24- Bennett SA, Beck JG, Clapp JD. Understanding the relationship between posttraumatic stress disorder and trauma cognitions: The impact of thought control strategies. Behav Res Ther. 2009;47(12):1018-23.

25- Berman NC, Wheaton MG, Fabriant LE, Jacobson SR, Abramowitz JS. The effects of familiarity on thought -action fusion. Behav Res Ther. 2011;49(10):695-9.

26- Besiroglu L, Çetinkaya N, Selvi Y, Atli A. Effects of selective serotonin reuptake inhibitors on thoughtaction fusion, metacognitions, and thought suppression in obsessive-compulsive disorder. Compr Psychiatry. 2011;52(5):556-61.

27- Berman NC, Wheaton MG, Abramowitz JS. Childhood trauma and thought action fusion: A multi-method examination. J Obsessive Compuls Relat Disord. 2013;2(1):43-7.

28- Ryff CD. Happiness is everything is it? Exploration on the meaning of psychological well-being. J Personal Soc Psychol. 1989;57(6):1069-81.

29- Bayani AA, Koocheky AM, Bayani A. Reliability and validity of ryff's psychological well-being scales. Iran J Psychiatry Clin Psychol. 2008;14(2):146-51. [Persian]

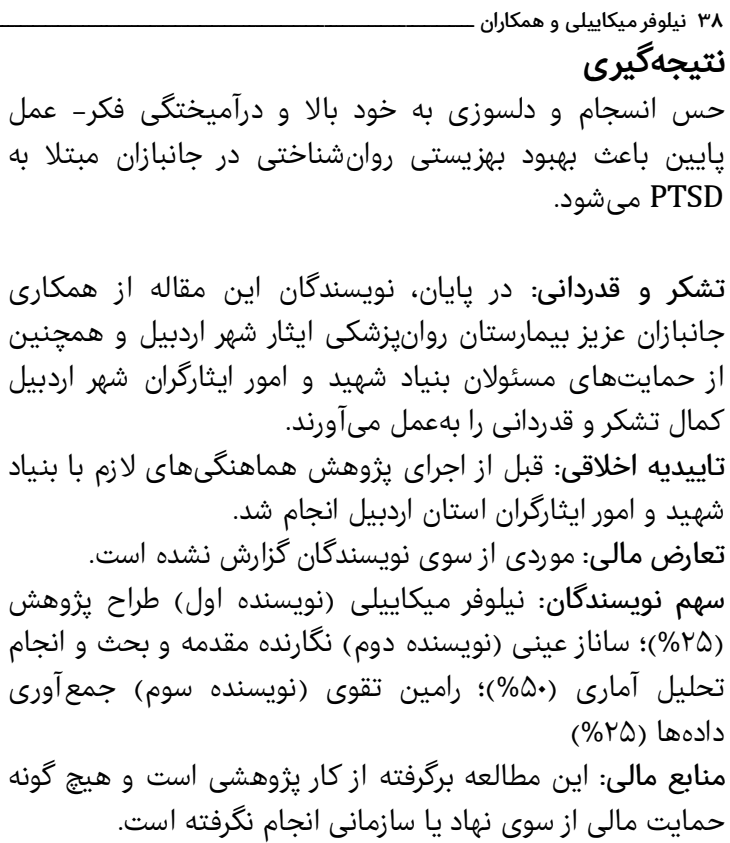

منابع

1- American Psychiatric Association. Diagnostic and statistical manual of mental disorders DSM-5. Rezaei F, Fakhraee A, charismatic A, lotus A, Hashemi Azar J, Shamloo F, translators. $5^{\text {th }}$ edition. Tehran: Arjmand Press; 2013. pp. 102-9. [Persian]

2- Karami GH, Amiri M, Ameli J, Kachuei H, Ghoddousi K, Saadat AR, et al. Psychological hcalth status of mustard gas cxposcd vctcrans. J Mil Med. 2006;8(1):1-7. [Persian] 3- Mohaghegh-Motlagh SJ, Momtazi S, Musavi-Nasab SN, Arab A, Saburi E, Saburi A. Post-traumatic stress disorder in male chemical injured war veterans compared to nonchemical war veterans. Med J Mashhad Univ Med Sci. 2014;56(6):361-8. [Persian]

4- Schultz M, Glickman ME, Eisen SV. Predictors of decline in overall mental health, PTSD and alcohol use in OEF/OIF veterans. Compr Psychiatry. 2014;55(7):165464.

5- Hebenstreit CL, Modden E, Koo KH, Maguen S. Minimally adequate mental health care and latent classes of PTSD symptoms in female Iraq and Afghanistan veterans. Psychiatry Res. 2015;230(1):90-5.

6- Sharifi T, Shokrkon H, Ahadi H, Mazaheri MM. The relationship between religious identity and national with students' mental health. J New Find Psychol. 2009;4(11): 125-42. [Persian]

7- Lareson JS. The measurement of health: Concepts and indicators. New York: Greenwood Press; 1991.

8- Ryff CD, Singer B. Human health: New directions for the next millennium. Psychol Inq. 1998;9(1):1-28.

9- Kelsall HL, Mc Kenzie DP, Forbes AB, Roberts MH, Urquhart DM, Sim MR. Pain-related musculoskeletal disorders, psychological comorbidity, and the relationship with physical and mental well-being in Gulf War veterans. Pain. 2014;155(4):685-92

10- Neff KD. Self-compassion: An alternative conceptualization of a healthy attitude toward oneself Self Identit. 2003;2(3):223-50.

11- Neff KD, Kirkpatrick KL, Rude SS. Self-compassion and adaptive psychological functioning. J Res Person. 2007;41(1):139-54. 
نقش دلسوزى به خود و حس انسجام و آميختى فكر- عمل در بيشبينى بهزيستى روان شناختى جانبازان مبتلا به اختلال استرس يس از سانحه و

Iranian sample. Procedia Behav Sci. 2010;5:1451-5.

33- Shafran R, Thordarson DS, Rachman S. Thoughtaction fusion in obsessive- compulsive disorder. J Anxiety Disord. 1996;10(5):379-91.

34- Pourfaraj M, Mohammadi N, Taghavi M. Psychometric properties of revised thought-action fusion questionnaire (TAF-R) in an Iranian population. J Behav Ther Exp Psychiatry. 2008;39(4):600-9.

30- Basharpoor S. Psychometric properties of the Persian version of the self compassion scale in university students. J Res Psychol Health. 2014;7(2):66-75. [Persian]

31- Antonovsky A. The structure and properties of the sense of coherence scale. Soc Sic Med. 1993;36(6):725-33.

32- Mahammadzadeh A, Poursharifi H, Alipour A. Validation of Sense of Coherence (SOC) 13- item scale in 